\title{
ENSINO DA LÓGICA NA FILOSOFIA: FILOSOFIA DA LÓGICA E PENSAR CRÍTICO
}

\author{
TEACHING LOGIC IN PHILOSOPHY: PHILOSOPHY OF LOGIC AND CRITICAL THINKING
}

Ralph Leal Heck*

\section{RESUMO:}

Este estudo busca uma caracterização da atividade de ensino da lógica que contemple o ensino da lógica enquanto elemento indispensável para o desenvolvimento da racionalidade humana; enquanto técnica para solução de problemas cotidianos; e enquanto locus de reflexões filosóficas frutíferas. Assim, dividiremos a argumentação em três partes. A primeira discute a competência fundamental para o sucesso da aprendizagem e aplicação prática da lógica, o pensar crítico. Para isto, seguiremos a definição de Jennifer W. Mulnix e outros autores (Vaughn, Willingham e Elder), pois entendem que a lógica se apresenta como condição necessária para o desenvolvimento e retroalimentação da competência do pensar crítico. A segunda expõe a estratégia de ensino da lógica na filosofia relatada por Sam Hiller, que julgamos ser útil, pois se ocupa em usar a lógica para desenvolver o pensar crítico, com ênfase na solução de problemas cotidianos. Na terceira parte, usando a distinção saber que e saber como, e a diferença epistemológica entre experiência da lógica e experiência na lógica, exploraremos elementos propriamente filosóficos, omitidos pelos autores citados, com o objetivo de apontar dois temas fundamentais, que completam o quadro de ensino da lógica na filosofia: os pressupostos epistêmicos da lógica e sua dimensão histórico-filosófica.

PALAVRAS-CHAVE: Lógica. Pensar Crítico. Ensino da Lógica. Filosofia da Lógica. Epistemologia da Lógica.

\begin{abstract}
:
This study aims to characterize the activity of teaching logic, which contemplates the teaching of logic as an indispensable element for the development of human rationality; as a technique for solving everyday problems; and as locus of fruitful philosophical reflections. To do so, we will divide the argument into three parts. The first one discusses the fundamental competence for the success of learning and practical application of logic, i.e. critical thinking. For this, we will follow the definition of Jennifer W. Mulnix and other authors (Vaughn, Willingham and Elder), who understand that logic presents itself as a necessary condition for the development and feedback of the competence of critical thinking. The second exposes the strategy of teaching logic in philosophy, reported by Sam Hiller, which we believe to be useful, because it is concerned with using logic to develop critical thinking, with an emphasis on solving everyday problems. In the third part, using the distinction between to know and to know how, and the epistemological difference between the experience of logic and experience in logic, we will explore properly philosophical elements, omitted by the authors mentioned, in order to point out two fundamental themes that complete the Teaching of logic in philosophy: the epistemic assumptions of logic and its historical-philosophical dimension.
\end{abstract}

KEY-WORDS: Logic. Critical Thinking. Teaching of Logic. Philosophy of Logic. Epistemology of Logic.

\footnotetext{
*Doutorando em Filosofia. Professor da Faculdade Católica de Fortaleza. E-mail: imagomundi@hotmail.com.
} 


\section{O CONHECIMENTO LÓGICO-MATEMÁTICO E O PENSAR CRÍTICO}

Neste primeiro tópico, veremos a principal competência necessária para efetivar o aprendizado dos conhecimentos e técnicas que se fundamentam no raciocínio lógicomatemático e que marcam negativamente os índices educacionais no cenário mundial, a saber: o pensar crítico. Em seguida, veremos como a lógica se relaciona com esse pensar crítico, por um lado, enquanto condição de desenvolvimento da habilidade e, por outro, enquanto domínio do conhecimento que viabiliza seu exercício. O objetivo deste tópico é demonstrar que o estudo e a aprendizagem da lógica implicam no desenvolvimento de uma competência vital para o gozo pleno da racionalidade humana ${ }^{1}$.

É fato que as sociedades contemporâneas, dotadas de uma enorme demanda profissional nas áreas de Ciência, Tecnologia, Engenharia e Matemática, se voltam para o público cada vez mais jovem. Mas, na contramão da exigência, desde muito cedo encontramos jovens com uma verdadeira fobia à matemática e à lógica, quando muito, uma formação precária, resultado de um ensino superficial e indiferente às técnicas pedagógicas que equacionem a dimensão teórica e prática desses conhecimentos.

É fato que países desenvolvidos possuem um histórico bem-sucedido em educação lógico-matemática (com ênfase em solução de problemas e abordagens conceituais) ${ }^{2}$. Nesses países, os estudantes são incentivados a embaterem-se com os problemas e resolvê-los por si mesmos, ao invés de serem conduzidos pelo professor, muitas vezes desejoso por ajudar no primeiro sinal de dificuldade. Note que, quando falamos em lógica e raciocínio lógicomatemático, somos remetidos inevitavelmente a uma longa tradição filosófica e matemática começando com Aristóteles ${ }^{3}$ até a contemporaneidade com Frege, Russell, Gödel, Carnap, Hilbert, Quine, Kripke, etc - que se ocupa em desenvolver a lógica (e a matemática) como um domínio de consideração filosófica (filosofia da lógica e filosofia da matemática) e como um conhecimento de utilidade prática. E segundo essa perspectiva prática, a principal competência que garante o sucesso do emprego da matemática (e não diferente, da lógica) é o

\footnotetext{
${ }^{1}$ Por exemplo, na Declaração dos Princípios da Tolerância da UNESCO, onde o pensar crítico é tido como uma das habilidades fundamentais para a paz e para o desenvolvimento econômico e social dos indivíduos. http://portal.unesco.org/en/ev.php-URL ID=13175\&URL_DO=DO TOPIC\&URL_SECTION=201.html .

2 Site da OECD, disponível em: http://www.oecd.org/pisa/data/; cf. Site com o ranque das melhores universidades do mundo em matemática, disponível em: http://www.usnews.com/education/best-globaluniversities/mathematics; Site do INEP contando os dados brasileiros do PISA, disponível em: http://portal.inep.gov.br/internacional-novo-pisa-resultados.

${ }^{3}$ Indo mais profundamente nas raízes do pensamento lógico, encontramos a fundação de seus conceitos em Platão e na tradição filosófica que o precedeu (MORAVCSIK, 2004, p.1-26).
} 
pensar crítico (critical thinking) ${ }^{4}$. Mas, o que é pensar crítico? Segundo Facione, Facione e Giancarlo (2000, p. 2):

O consenso geral é que o pensamento crítico (CT) per se é julgar de forma reflexiva o que fazer ou no que acreditar. As habilidades cognitivas de análise, interpretação, inferência, explicação, avaliação e de monitoramento e correção do próprio raciocínio são o cerne do pensamento crítico [...]. (tradução nossa).

Esses mesmos autores definem duas classes de características que compõem o conceito de pensar crítico: as habilidades (skills) e as disposições (dispositions) (uma ativa e uma passiva, respectivamente). As habilidades são as capacidades de executar: análise, inferência, explicação, interpretação, auto-avaliação e valoração, compostas de diversas sub-habilidades ${ }^{5}$. E entre as disposições estão: disponibilidade à verdade, mente aberta, analiticidade, sistematicidade, autoconfiança em seu pensar crítico, inquirição e maturidade de julgamento (FACIONE; FACIONE; GIANCARLO, 2000, p. 21-23).

Mas, como bem aponta Mulnix, o tipo de definição acima é muito amplo e pode levar a julgamentos equivocados e até contraditórios sobre o que exatamente é o pensar crítico. Sendo assim, elencaremos duas definições menos abrangentes que dispõem o raciocínio lógico e prático como o centro da definição do termo. A primeira:

O Pensar Crítico [é] a avaliação ou formulação sistemática de crenças, ou declarações, através de padrões racionais. O pensar crítico é sistemático porque envolve procedimentos e métodos distintos [...] E opera de acordo com padrões racionais em que as crenças são julgadas por quão bem elas são apoiadas por razões. (VAUGHN, MACDONALD, 2007, p.4, tradução nossa).

A segunda definição:

[...] o pensar crítico consiste em ver os dois lados de uma questão, estando aberto a novas evidências que desconfirmem suas ideias, raciocinando desapaixonadamente, exigindo que as reivindicações sejam apoiadas por evidências, deduzindo e inferindo conclusões a partir de fatos disponíveis, resolvendo problemas e assim por diante. (WILLINGHAM, 2014. p. 8) (tradução nossa)

Estas duas definições se diferenciam pelo papel que executam na compreensão da categoria pensar crítico enquanto ação humana racionalmente mediada. Como aponta Mulnix

\footnotetext{
${ }^{4}$ Suas origens também podem ser traçadas até Platão e Aristóteles. No que compete à relação entre retórica e lógica. Para uma caracterização desta origem e sua aplicação no contexto do ensino superior (ANDREWS, 2015. pp.49-62).

5 De fato, são reconhecidas as 6 habilidades citadas, 16 sub-habilidades e 19 disposições (ABRAMI, BERNARD, BOROKHOVSKI, WADEM, SURKES, TAMIM, ZHANG, 2008, p. 1102-1134).
} 
(MULNIX, 2010, p. 5-6), a diferença entre elas é de "saber que" e "saber como". A primeira definição evidencia a compreensão sistemática e procedural da atividade de pensar, permitindo ao indivíduo reconhecer regras e etapas do processo - inclusive seus limites, vantagens e implicações meta-teóricas. A segunda definição evidencia a atividade prática, a capacidade de resolver problemas, na medida em que o sujeito se confronta com eles. Mulnix sintetiza essas perspectivas na seguinte definição para o pensar crítico:

O pensar crítico é um processo, uma atividade especializada do pensamento. Inclui o compromisso em usar a razão na formulação de nossas crenças. Não é o mesmo que pensar criativamente, imaginativamente ou baseando-se na emoção. E, assim como em qualquer habilidade, ela pode ser possuída em maior ou menor grau. (MULNIX, 2010. p.8) (tradução nossa)

Mas, como ensinar o pensar crítico? Alguém poderia argumentar que a maior parte dos seres humanos raciocina logicamente sem qualquer instrução em lógica. De fato, ensinar o raciocínio crítico não envolve auxiliar na obtenção dos resultados das habilidades de inferência e formulação de hipóteses, por exemplo. Mas, sim, ensinar essas habilidades $e$ levar os indivíduos a explicar como eles resolveram os problemas, segundo seu contexto de resolução, enquanto discutem suas ideias com os outros. Com isso, os indivíduos se tornam capazes de resolver problemas enquanto são ensinados a analisar analogias; criar categorias e classificar itens; identificar informação relevante; construir e reconhecer argumentos dedutivos válidos; testar hipóteses; reconhecer falácias do senso comum; distinguir entre evidências e interpretações de evidências, tudo isso, sozinhos ou em grupo ${ }^{6}$, além de ter disposições incentivadas como curiosidade, flexibilidade e mente-aberta (ABRAMI, 2008, p. 328). Nesse sentido, o domínio da lógica se insere como condição de possibilidade do pensar crítico.

\subsection{Importância da lógica no pensar crítico}

A prova da importância da lógica em nossas vidas está no uso que fazemos das habilidades de análise e inferência para solucionar problemas cotidianos, como elaborar um

\footnotetext{
${ }^{6}$ Segundo a American Philosophical Association, há algumas recomendações que auxiliam no desenvolvimento do pensar crítico em crianças, mas que podem ser adaptadas ao cenário do ensino superior. São elas: 1.Ensinar aos jovens a dar justificativas para suas conclusões; 2.Pedir que deem razões para a execução de certos comandos e comportamentos; 3.Encorajar os jovens a fazer perguntas.

4.Pedir aos jovens que elaborarem soluções e explicações alternativas para um mesmo problema. 5. Pedir que esclareçam o significado dos termos que empregam. 6. Conduzir debates sobre preconceitos. 7. Não restringir o raciocínio crítico ao âmbito estritamente empírico ou acadêmico-conceitual. 8. Exigir que os jovens a escrevam. (FACIONE, 1990.)
} 
orçamento doméstico, que faça sobrar algum dinheiro no fim do mês; fazer uma lista de compras com produtos que durem por trinta dias; preparar um jantar para quatro pessoas, em 50 minutos, apenas com os ingredientes que você possui em casa; ou até mesmo aproveitar uma promoção de frete grátis em uma loja online, tudo isso, sendo capaz de explicitar cada etapa desenvolvida no raciocínio. Os julgamentos que envolvem cada uma das atividades descritas são da mesma natureza que os procedimentos dedutivos em sistemas lógicos. De fato, para ainda outros autores, a lógica é a essência do pensar crítico:

Através do pensar crítico, como eu o entendo, adquirimos um meio de avaliar e aprimorar nossa capacidade de julgar bem. Ela nos permite entrar em virtualmente qualquer situação e descobrir a lógica do quer que seja que esteja acontecendo nessa situação. Ele fornece uma maneira de aprendermos com novas experiências através do processo contínuo de auto avaliação. O pensar crítico, então, nos permite formar sólidas crenças e julgamentos e, ao fazê-lo, nos fornece uma base para uma vida emocional "racional e razoável". (ELDER, 1996, p. 38, tradução e grifo nossos).

Dedicando igual importância à lógica como fundamento do pensar crítico, Mulnix defende que:

\begin{abstract}
Raciocinar bem, ou pensar criticamente, consiste primeiramente na capacidade de compreender conexões inferenciais entre declarações, a fim de ver uma progressão da evidência em forma de argumento em direção a uma conclusão específica. Ser um pensador crítico proficiente, então, é ver claramente a relação entre evidência e conclusão, e ser proficiente em fornecer razões em apoio às crenças. Isso deve incluir, também, a capacidade de reconhecer o que seria considerado evidência contra as crenças. (MULNIX, 2010, p.10, tradução e grifo nossos).
\end{abstract}

Mas, antes, devemos nos perguntar o que significa lógica. Para Frege, um dos fundadores da lógica-matemática e da filosofia analítica da linguagem “descobrir verdades é a tarefa de todas as ciências: cabe à lógica, porém, discernir as leis do ser verdadeiro (Wahrsein) (FREGE, 2002, p. 11)”. Uma definição com forte apelo semântico. Já para Cook (2009, p. 174), "lógica é o estudo de argumentos e dos vários métodos lógicos usados para investigar a estrutura de argumentos e para classificá-los, em termos de sua estrutura, aqueles que devem ser persuasivos e aqueles que não devem ser persuasivos"; uma definição com ênfase na epistemologia da lógica. Enquanto para Tarski (TARSKI, 1994, p. xii), "a palavra 'lógica' é usada [...] como o nome de uma disciplina que analisa o significado dos conceitos comuns a todas as ciências e estabelece as leis gerais que governam estes conceitos"; uma definição semântico-extensional. De modo informal, podemos resumir que a Lógica são conjuntos de técnicas que visam raciocínios persuasivos por força da verdade que eles 
preservam em cada etapa da cadeia de um raciocínio, a partir de suas premissas, com vistas a preservar certas propriedades dos argumentos como consistência, coerência, etc. Ou melhor, a lógica é o estudo de certos sistemas de inferências, a partir de onde podemos derivar certas afirmações (conclusões) a partir de outras (premissas) (IMAGUIRE; BARROSO, 2006, p. 11). Com essas definições, não é difícil ver que as habilidades de análise, inferência, interpretação e auto-avaliação, bem como, as disposições de analiticidade e sistematicidade necessárias ao pensar crítico são implicadas pelo conhecimento da lógica ${ }^{7}$.

\section{O PENSAR CRÍTICO ATRAVÉS DA LÓGICA}

Neste tópico, faremos uma análise dos objetivos da aquisição do pensar crítico, orientado ao conhecimento da lógica. Nesse sentido, iniciaremos com uma avaliação crítica da proposta de Mulnix e utilizaremos a proposta pedagógica de ensino da lógica de Hiller para resolver lagumas lacunas deixadas por ela (a saber, as questões (M1), (M2) e (M3)), mas, que deixam intocados certos temas vitais para o ensino da lógica, em especial, na filosofia (a saber, as questões (H1), (H2), (P1) e (P2)).

Como vimos, Mulnix nos oferece um modelo geral de ensino do pensar crítico através da lógica. Esse modelo é dotado de propriedades valiosas para satisfazermos as exigências levantadas no final do tópico anterior, contudo carece de elementos práticos que o tornam insuficiente para o objetivo a que se propõe, como será visto adiante.

Para ela, o ensino do pensar crítico deve (MULNIX, 2010. p.11):

(i) Conduzir o estudante a um estado meta-cognitivo de consciência de seus processos de pensar.

(ii) Todo curso que se proponha a desenvolver o pensar crítico nos estudantes deve ter como fundamental preocupação ensiná-los a captar (to grasp) as conexões inferenciais e evidenciais dos argumentos.

(iii) $\mathrm{O}$ que implica reconhecer entre as evidências usadas em um argumento e a conclusão ao qual o argumento fornece suporte.

(iv) Satisfeitas as exigências acima, o estudante deve conhecer a teoria do pensar crítico. O porquê de seu modo de pensar ser importante e quais explicações dão importância para este modo de pensar. Este tópico é o "saber que" [know that] apresentado no início do texto.

\footnotetext{
${ }^{7}$ Para o caso do ensino da lógica, Cothran, sugere três regras, considerando todos os seguintes sub-tópicos da lógica: lógica formal, composta de raciocínio dedutivo e indutivo, e lógica informal (material) composta da retórica, sofística (falácias) e solução de problemas. As regras são: 1. Ensine o raciocínio correto primeiro, as falácias (raciocínio incorreto) depois. 2. Ensine lógica formal e, depois, lógica informal. 3. Estude dedução antes de indução (COTHRAN, https://www.memoriapress.com/articles/how-teach-logic/).
} 
Para solucionar (i), exercícios sobre pensar crítico devem ser executados e reiterados substancialmente pelo estudante. Os objetivos (ii) e (iii) são resolvidos, segundo a autora, com o uso de mapeamento de argumentos ${ }^{8}$. Nas palavras de Mulnix:

\begin{abstract}
As cadeias de raciocínio e evidência estão estruturadas hierarquicamente, com algumas premissas apoiando outras e fornecendo apoio à conclusão, seja indiretamente, conjuntamente ou independentemente, tal que podemos diagramar essas estruturas. [...] Não deve ser surpreendente que esta técnica [de mapeamento de argumentos] ajude a estimular as habilidades de raciocínio crítico, uma vez que concentra a atenção do aluno sobre as conexões inferenciais entre as declarações e como algumas declarações podem trabalhar em conjunto, em uma variedade de modos, a prestar apoio como evidências a outras declarações. (MULNIX, 2010, p. 12 , tradução nossa)
\end{abstract}

Para solucionar (iv), ela sugere que as etapas (i), (ii), e (iii) devam ser satisfeitas, para então falar da teoria do pensar crítico, mas não esclarece de que modo e qual teoria deve ser ensinada. Com efeito, o maior problema parece ser o fato de a autora não oferecer uma proposta metodológica ou pedagógica de emprego da lógica para o desenvolvimento do pensar crítico, salvo o mapeamento de argumentos, que corresponde a apenas um momento do longo percurso de aprendizagem da lógica.

Há, em suma, uma distinção epistemológica valiosa e duas intuições teóricas interessantes no argumento de Mulnix que, infelizmente, não são levadas adiante:

- (M1) A distinção entre "saber que" [know that] e "saber como" [know how].

- (M2) O estado meta-cognitivo do sujeito do pensar crítico.

- (M3) O ensino da teoria lógica do pensar crítico.

(M1) distingue o papel do conhecimento acerca do pensar crítico, se ele é um conhecimento enquanto representação de fatos [know that], expressão de uma dada teoria (i.e. expresso através da função descritiva da linguagem) ou se ele é uma prática executável, sempre que se faz necessário, através de certo contexto [know how]. Essa distinção pode ser empregada para qualquer atividade humana; com especial valor, se empregada na lógica, que deve ser o suporte para se alcançar o conhecimento, em duplo sentido, do pensar crítico. Entretanto, a autora se restringe à distinção no âmbito do pensar crítico sem tematizar seus pressupostos sob essa ótica. Para (M2), infelizmente, a autora não fornece nenhuma definição

\footnotetext{
${ }^{8}$ Um mapa de argumento [argument mapping] é a construção em grafo das afirmações das premissas de uma dada argumentação textual (livro, revista, discurso, jornal etc.) [extended argument] e o modo como as premissas se relacionam e suportam a conclusão do argumento. Há diversos padrões de argumentos: conclusão múltipla, premissas conjuntas, padrão horizontal, padrão vertical etc. (HURLEY, 2012, p. 64-75).
} 
do termo. Em (M3), Mulnix se limita a fazer analogias sobre o efeito pouco relevante do conhecimento representacional no ensino do pensar crítico, como levar um tenista iniciante para assistir uma partida de Wimbledon esperando que ele saia da partida sabendo tanto quanto os jogadores, ou um golfista iniciante ser levado a ler um artigo na Golf Digest para melhorar suas tacadas. Ou mesmo, fazer o download da informação (no estilo do filme Matrix) sobre um assunto, sem ter realizado prática alguma com ele. Os pontos (M1), (M2) e (M3) serão retomados no início da próxima sessão.

Quanto à ausência da proposta metodológica, encontramos em Sam Hiller (2014, p. 1936), uma forma de equacionar a aprendizagem das técnicas formais da lógica, sua aplicação prática e o desenvolvimento do pensar crítico. Ele o faz a partir da descrição de sua experiência de ensino da disciplina Lógica prática (Pratical logic), que corresponde aos temas tradicionalmente ensinados nos cursos de lógica para a filosofia: lógica dedutiva, lógica indutiva, raciocínio causal, teoria da probabilidade, falácias, preconceitos cognitivos, o método científico, e raciocínio criativo. Com a diferença que é orientada pelos princípios de bom raciocínio (good reasoning) (isto é, raciocínio debatido e justificado), conclusões por criação de alternativas (técnicas combinatórias ou conjecturas criativas) e eliminação de alternativas (por dedução e verificação de inconsistências). Em cada um dos tópicos propostos, Hiller apresenta uma abordagem técnica distinta, com o objetivo de abranger a maior quantidade de conteúdo formal combinado com a prática. Vejamos cada um deles.

O primeiro tópico é sobre lógica dedutiva; o autor sugere utilizar como ponto de partida os problemas LSAT $^{9}$. Eles devem ser resolvidos usando-se os instrumentais clássicos da lógica proposicional (LP), avaliando-se as possibilidades e resultados de combinações de valores de verdade de sentenças proposicionais, representando situações de combinação dos elementos do problema. Dadas as premissas, os estudantes devem eliminar as possibilidades inconsistentes e encontrar uma ou mais respostas admissíveis. Por exemplo:

\footnotetext{
O gerente de vendas Phil Forrester está tentando montar uma equipe de vendas para cobrir a área de Los Angeles. Sua equipe será composta por quatro membros - dois experientes e dois novos vendedores.

Sam, Fred, Harry e Kim são os vendedores experientes.

João, Tim e Dom são novos.

Sam e Fred não trabalham juntos.

Tim e Sam se recusam a trabalhar juntos.

Harry e Dom não podem trabalhar juntos.
}

\footnotetext{
9 LSAT significa "Law School Admission Tests". É um teste para avaliar interpretação textual e raciocínio lógico, usualmente empregado como processo de admissão em cursos de Direito nos EUA e Canadá.
} 
Começo contando os arranjos possíveis. Existem 4 × 3 × 3 X $2=72$ equipes possíveis, mas as três premissas fornecidas na questão trabalham para eliminar um certo número dessas alternativas. (HILLER, 2014, p. 26, tradução nossa).

Diante desse cenário, ele levanta a seguinte pergunta: se Dom é escolhido como parte da equipe de vendas, mas John não o é, então, os outros três membros devem ser: a) Fred, Tim, e Harry; b) Fred, Tim, e Kim; c) Harry, John, e Tim; d) Tim, Dom, e Kim; e) Sam, Fred, e Harry. Com a adição da condicional no enunciado, John está fora do grupo e também Harry (por causa de Dom). Como um dos postulados diz que deve haver dois membros novos, então necessariamente Tim faz parte do grupo. Mas, se ele faz parte, então Sam está fora. Restando apenas uma possibilidade: Dom, Tim, Fred e Kim, o item (b).

A associação dos problemas em LSAT com a lógica proposicional ocorre em função da combinação de situações possíveis. Onde cada indivíduo (Sam, Fred, Harry, Tim, Kim, John, Dom) está para uma proposição (uma letra proposicional: s, f, h, t, k, j, d) e cada conjunto de combinações entre eles corresponde a uma situação possível, um mundo possível ${ }^{10}$. A ideia de Hiller é:

\begin{abstract}
Usar tabelas de verdade para mostrar conexões sentenciais e interpretar cada linha de uma tabela de verdade como um mundo possível diferente. Argumentos na lógica sentencial podem então ser pensados de uma maneira similar aos problemas LSAT: há um número de alternativas possíveis e tonar cada premissa verdadeira, funciona eliminando alternativas (aquelas onde essa premissa é falsa). Depois de satisfazer todas as premissas, verifique se a conclusão é verdadeira em cada linha remanescente. Se for falso, então há um contraexemplo: uma alternativa não eliminada, que mostra que o raciocínio presente no argumento não é válido. Caso contrário, o argumento é dedutivamente válido: se as premissas forem verdadeiras, então a conclusão deve ser verdadeira. Só depois disso, lhes dou a lista padronizada de tipos de argumentos válidos (modus ponens, silogismo hipotético, etc.). (HILLER, 2014, p. 24, tradução nossa).
\end{abstract}

O segundo tema apresentado é Lógica Indutiva. Normalmente, a indução lógica funciona da seguinte forma: seja $\mathrm{x}_{1}$ um objeto com a propriedade $\mathrm{P}, \mathrm{x}_{2}$ um objeto com a propriedade $\mathrm{P}, \ldots, \mathrm{x}_{\mathrm{n}}$ um objeto com a propriedade $\mathrm{P}$, então todos os objetos $\mathrm{x}$ possuem a propriedade P. Hiller propõe que a indução sobre propriedades e classes seja feita por plausibilidade de alternativas: "o quão plausível é o caso de um caso de objeto x não ter a

\footnotetext{
${ }^{10}$ Embora Hiller sugira a abordagem com as tabelas de verdade, no texto não se explica em que momento da resolução do problema deve ser introduzida a tabela. Note que são sete proposições elementares, portanto um total de 128 linhas! Inexequível em sala de aula. Minha sugestão é realizar via dedução uma redução de possibilidades e então aplicar a tabela no enunciado do problema. Por exemplo, como Dom é exigido no grupo e John é excluído dele, suas possibilidades de combinação não são computadas e no lugar delas marca-se com uma constante proposicional de valor pré-definido (T para Dom e F para John), igualmente para Tim (T) que é o único restante no grupo dos vendedores novos. Restando apenas 4 nomes e três premissas para se avaliar. Uma tabela razoável de 16 linhas.
} 
propriedade P?". Isto é, os estudantes são encorajados a imaginar/criar situações possíveis em que os antecedentes são verdadeiros e a conclusão é falsa. Além disso, eles procuram inferir os resultados de uma indução através da melhor explicação possível, questionando-se as causas de determinada consequência inesperada. A condução do professor, nesse caso, consiste em levantar com os estudantes uma lista de possíveis causas e eliminar uma a uma. iniciando-os no método científico e incentivando-os a desenvolver a criatividade necessária para o pensar crítico.

O terceiro tema é Raciocínio Causal, uma forma de continuação do tópico anterior. Hiller sugere iniciar com a diferença entre condição necessária e condição suficiente (associando a discussão com as proposições condicionais - implicação material - da lógica dedutiva), em seguida, caracterizar os métodos de concordância e diferença de John Stuart Mill (Method of agreement; Method of difference) ${ }^{11}$ :

1. Uma propriedade que está ausente quando o efeito está presente não pode ser uma condição necessária para esse efeito (Método de Concordância).

2. Uma propriedade que está presente quando o efeito está ausente não pode ser uma condição suficiente para esse efeito (Método de diferença). (HILLER, 2014, p. 26, tradução nossa).

Como o método de Mill serve para eliminar alternativas, é necessário recorrer à criatividade para elaborar uma lista inicial de possíveis causas. Se o método proposto pelo professor eliminar todas as causas, novas causas devem ser elaboradas pelos estudantes. Caso a lista não satisfaça a explicação causal, lembre-os de que esse problema é um dos principais argumentos contra a indução empírica como condição necessária ou suficiente para a elaboração de leis científicas. Para exercitar a reflexão, Hiller sugere a investigação de casos cotidianos, até mesmo de casos atípicos como nas estórias de Sherlock Holmes e Dr. House (o seriado de TV).

No tópico sobre Probabilidade, ao invés de usar o método tradicional de cálculo axiomático-dedutivo bayesiano de probabilidade (COX, 2001.), Hiller sugere um pequeno sistema de regras que segue o mesmo comportamento do cálculo bayesiano envolvendo fórmulas proposicionais, fundamentos da teoria da informação semântica e diagramas de Venn, todos com o objetivo de tornar a compreensão dos princípios do cálculo de probabilidade mais intuitiva. Nesse método, cada sentença proposicional ocupa determinado

\footnotetext{
11 O primeiro método avalia se ocorre uma bi-implicação entre a Propriedade e o Efeito. O segundo método avalia se a Propriedade implica materialmente o Efeito.
} 
espaço em um diagrama de Venn de distribuição de probabilidades. A cada introdução de informação nova, a distribuição das probabilidades é atualizada, modificada e um conjunto de alternativas é descartado. As regras que orientam o sistema são:

1. Regra limítrofe: para toda sentença $\mathrm{Q}, 0 \leq \operatorname{Pr}(Q) \leq 1$, onde 0 é a menor informação veiculável, à saber, nenhuma (não há informação negativa neste modelo).

2. Regra da tautologia: Se $Q$ é uma tautologia $\operatorname{Pr}(Q)=1$, isto é, ela é verdadeira em todo espaço de probabilidades, portanto, ocupa todo o espaço de distribuição disponível.

3. Regra da contradição: Seja $Q$ uma contradição, então $\operatorname{Pr}(Q)=0$. Portanto, não ocupa espaço na área do diagrama.

4. Regra da negação: Para toda sentença $Q, \operatorname{Pr}(\neg Q)=1-\operatorname{Pr}(Q)$, ou seja, a negação de uma sentença é o complemento da área de probabilidade da sentença.

5. Regra da equivalência lógica: Sejam P e Q logicamente equivalentes, então $\operatorname{Pr}(P)=\operatorname{Pr}(Q)$, portanto, ocupam o mesmo espaço no diagrama (são coextensionais no espaço de probabilidade), representado na lógica proposicional como $P \leftrightarrow Q$.

6. Regra da Exclusão mútua: Sejam $\mathrm{P}$ e $\mathrm{Q}$ mutuamente excludentes, então, $\operatorname{Pr}(P \wedge Q)=0$. Portanto, não há sobreposição entre as áreas de $\mathrm{P}$ e $\mathrm{Q}$.

7. Regra da Disjunção: Para quaisquer sentenças $\mathrm{P}$ e $\mathrm{Q}, \operatorname{Pr}(P \vee Q)=\operatorname{Pr}(P)+$ $\operatorname{Pr}(Q)-\operatorname{Pr}(P \wedge Q)$. Esta regra cria um novo espaço combinando os espaços de probabilidade de $\mathrm{P}$ e Q, sem errar ao duplicá-los caso haja sobreposição.

8. Regra da Disjunção especial: Para quaisquer sentenças $P$ e $Q$, se elas são mutuamente excludentes, então sua disjunção $\operatorname{Pr}(P \vee Q)=\operatorname{Pr}(P)+\operatorname{Pr}(Q)$. O que é obvio, pois $\operatorname{Pr}(P \wedge Q)=0$.

9. Regra da Conjunção: Para quaisquer sentenças $\mathrm{P}$ e $\mathrm{Q}, \operatorname{Pr}(P \wedge Q)=\operatorname{Pr}(P) \times$ $\operatorname{Pr}(Q \mid P)$. Onde $\operatorname{Pr}(Q \mid P)$ é a probabilidade condicional $P \rightarrow Q$, representado no diagrama como a área sobreposta entre $\mathrm{P}$ e Q. Assim, o cálculo avalia a área de $\mathrm{P}$ multiplicado pela área de $\mathrm{Q}$, caso $\mathrm{P}$ seja verdadeiro. Note que $\operatorname{Pr}(Q \wedge P)=$ $\operatorname{Pr}(Q) \times \operatorname{Pr}(P \mid Q)$ gera a mesma área que a fórmula anterior. 
10. Regra da conjunção especial: para o caso em que P e Q sejam independentes, $\operatorname{Pr}(P \wedge Q)=\operatorname{Pr}(P) \times \operatorname{Pr}(Q)$

No tópico seguinte, Hiller apresenta o método científico. De fato, esse assunto é uma extensão da indução lógica e do raciocínio dedutivo cotidiano, com um toque de falsificacionismo. Para exemplificar o tema pede-se que seja gerada uma lista de possíveis causas $\mathrm{X}$ para um evento/fenômeno $\mathrm{Y}$, em um dado grupo experimental. Usando os critérios de Mill, fazemos as eliminações das causas de Y, em seguida, verificamos os resultados em um grupo de controle. Da perspectiva do cálculo bayesiano (do grau de informatividade e certeza), se uma predição E pode ser deduzida de uma hipótese $\mathrm{H}$ como um argumento dedutivamente válido, então: $\operatorname{Pr}(H \mid \neg E)=0$ e $\operatorname{Pr}(H \mid E)>\operatorname{Pr}(H)$.

O tópico seguinte é sobre falácias. Nele, é apresentado um conjunto de falácias interpretadas sob os problemas da geração de alternativas irrelevantes (e erradas) e da eliminação de alternativas corretas:

Algumas falácias lógicas, como Afirmando o Consequente, Generalização Apressada, ou Ad Hominem, não conseguem eliminar alternativas relevantes. Outros, como a Falsa Dicotomia ou "Bola de Neve", cegam aquele que raciocina à existência de outras possibilidades. Em ambos os casos, as falácias são erros na forma básica do bom raciocínio (good reasoning) como gerador e eliminador de alternativas. (HILLER, 2014, p. 30, tradução e grifo nossos).

O tópico seguinte é preconceitos cognitivos. A proposta é que o estudante capaz de empregar o raciocínio lógico e o pensar crítico saiba lidar satisfatoriamente com preconceitos psicológicos. A justificativa é que esses preconceitos nos cegam para alternativas relevantes, nos levando a ir à conclusão quando ainda restam alternativas. O objetivo é que os estudantes sejam capazes de identificar um raciocínio dogmático, não refletido. O exemplo proposto é o exercício de identificação de sequências numéricas. Uma sequência feita com regras gerais permite muitas interpretações de sequências criadas a partir de regras restritas, estando todos os exemplos criados a partir das interpretações verdadeiras. O método de contraexemplo eficiente é executar um alargamento gradual das regras até que um exemplo derivado da regra suposta não seja aceito. A partir daí, testar as aproximações da regra original até que se chegue à resposta certa.

O último tópico é o pensar criativo. Hiller propõe dois métodos para empregar o pensar criativo no raciocínio lógico. Um método positivo, que visa provar que dado argumento é forte, ele consiste em demonstrar que as premissas eliminam todas as 
alternativas, salvo a conclusão. E um método negativo, que visa provar que o argumento não é fraco, o método consiste em demonstrar que não há contraexemplos em que as premissas são verdadeiras e a conclusão é falsa.

Ora, a preocupação de Hiller é transmitir a lógica através de situações-problema do cotidiano. De fato, uma forma de ensinar a lógica enquanto desenvolve-se o pensamento crítico, tendo como pressuposto duas noções filosóficas interessantes, em primeiro lugar, que chamaremos de (H1), a ideia de que sua concepção de dedução é transformacional (ocupada exclusivamente em localizar inconsistências entre as hipóteses e a conclusão) no lugar da noção de dedução clássica (ocupada em garantir a verdade das premissas na conclusão). É essa diferença que Corcoran nomeia de filosofia da lógica teorético-transformacional e filosofia da lógica teorético-informacional (CORCORAN, 1999, p. 27). Em segundo lugar, que chamarei de (H2), o método falsificacionista clássico de Popper (1972), i.e. iniciar o procedimento de dedução contando com um conjunto previamente elaborado de hipóteses, que devem ser testadas logicamente e empiricamente (claro que, no contexto do ensino de lógica, a parte empírica pode ser descartada).

Embora a competência na lógica seja um pressuposto para o desenvolvimento do pensar crítico, como atesta Hiller, a ênfase em seu aspecto formal, tal qual é apresentado em manuais de lógica, não nos parece capaz de desenvolver eficientemente o pensamento crítico, especialmente se pensarmos no contexto brasileiro em que o ensino da lógica na filosofia costuma ocorrer em duas, em raros casos, três disciplinas (Lógica I, Lógica II e Lógica III) e os conteúdos, quando dedicados exclusivamente ao aspecto formal, costumam dividir-se entre: teoria dos conjuntos, lógica proposicional e teoremas (Lógica I), lógica de primeira ordem e teoremas (Lógica II), em alguns casos (com Lógica III), lógica modal alética, lógica epistêmica e/ou teoria dos modelos.

Alguns aspectos do estudo da lógica, em especial os temas abordados nos cursos de filosofia não são suficientemente contemplados. O estudo da lógica no contexto dos cursos de graduação em filosofia no Brasil (quando não possuem ofertas de disciplinas específicas para cada tema) deve equacionar três principais eixos: a história da lógica, as técnicas de lógica e a filosofia da lógica. A história da lógica corresponde ao percurso que a lógica fez no Ocidente desde sua primeira sistematização com Aristóteles até a atual condição pluralista (de sistemas não clássicos e extensões de clássicos) que toma conta do cenário mundial, de modo que o indivíduo esteja consciente do contexto históricop de determinado sistema. As técnicas da lógica devem contemplar os métodos de dedução, análise e a metalógica própria de cada 
sistema lógico. A filosofia da lógica, por sua vez, corresponde ao levantamento e exame crítico das consequências de certos metateoremas e das consequências da adoção de dado sistema em outras disciplinas filosóficas, por exemplo, as consequências epistemológicas da adoção de uma dada lógica paraconsistente ou as consequências metafísicas da adoção de um dado sistema de lógica modal, digamos S4. E esses são dois problemas que não foram resolvidos, sequer tematizados, por Mulnix, nem por Hiller: (P1) o problema da Filosofia da lógica e (P2) O problema da História da Lógica. Isto é, ainda que seja possível executar o programa da Lógica Prática, restariam duas questões capitais para a compreensão e competência da lógica e do pensar crítico: (P1) corresponde à pergunta pelas consequências (extra-pragmáticas, em longo prazo, etc.) da adoção de determinado sistema lógico, ou determinadas propriedades lógicas em um raciocínio, isto é, quando nos perguntamos qual o sentido de certos fatos sobre a lógica. Esse nível de indagação evidencia os comprometimentos implícitos contidos na adoção de determinada lógica, ou princípios lógicos. Tal âmbito de questionamento exige a elaboração de uma distinção entre experiência da lógica e experiência na lógica, que veremos adiante. (P1) nos permite evidenciar os comprometimentos metafísicos e epistemológicos implícitos em um sistema lógico (MADDY, 2014, p. 93-108); já (P2) corresponde à compreensão do contexto e das forças históricas que deram origem (e que indicam contextos práticos de aplicabilidade) a determinado sistema ou propriedade lógica. Julgar determinado tipo de dedução, estratégia de prova ou sistema lógico, como o mais adequado para a resolução de um problema em um dado contexto, exige do estudante mais que a competência em executar ou apresentar soluções, mas antes a capacidade de reconhecer a origem e a finalidade dos elementos que formam o cenário do problema. Uma compreensão elementar em história da lógica nos fornece a teoria para empreender a leitura desses contextos.

\section{UNIFICANDO PERSPECTIVAS: A EXPERIÊNCIA NA LÓGICA E A EXPERIÊNCIA DA LÓGICA}

Neste último tópico, retomaremos todas as questões deixadas em aberto nos tópicos precedentes e buscaremos solucioná-las com o objetivo de fornecer uma visão mais completa possível das abordagens necessárias ao ensino da lógica (a técnica da lógica, a prática da lógica, a história e a filosofia da lógica), tendo em vista o desenvolvimento do pensar crítico.

Vamos iniciar com a lista de questões que foram delineadas ao longo de nossa exposição: 
- (M1) O primado epistemológico do "saber como" [know how $]$ sobre o "saber que" [know that $]$ na aprendizagem do pensar crítico.

- (M2) O estado meta-cognitivo não esclarecido do sujeito do pensar crítico.

- (M3) A ausência de apresentação de uma teoria lógica sobre o pensar crítico.

- (H1) No ensino do pensar crítico através da lógica proposto por Hiller, a implicação de uma dedução difere do significado clássico de preservação da verdade por transferência de informação.

- (H2) O primado da criatividade e da busca por inconsistências em hipóteses. aplicado á lógica demandam esclarecimento da relação entre conhecimento lógico e raciocínio ordinário.

- (P1) O problema da Filosofia da Lógica

- (P2) O problema da História da Lógica.

Vamos começar esta seção encontrando uma definição para o estado meta-cognitivo, a questão (M2). Segundo o Dictionary of Cognitive Science (HOUDÉ, 2004. pp.259-262.), o estado meta-cognitivo (ou meta-cognição) é composto por duas dimensões: o conhecimento meta-cognitivo e as experiências meta-cognitivas. O primeiro, chamado também de metaconhecimento (metaknowledge), é um conjunto de conhecimentos e crenças armazenados na memória de longo prazo sobre fatores que afetam o progresso e o resultado de processos cognitivos e tem como objeto pessoas, tarefas e estratégias. Apesar de ser um conhecimento de segunda ordem, ele não difere qualitativamente de outros tipos de conhecimento. Neste sentido, o meta-conhecimento é um conhecimento representacional (proposicional) acerca de conhecimentos e crenças.

Já as experiências metacognitivas são todas as experiências afetivas e cognitivas conscientes, ligadas à resolução de um problema particular:

\footnotetext{
Essas experiências transitórias ocorrem especialmente quando o sujeito está executando uma tarefa cognitiva relativamente complexa - isto é, complexa para o nível de desenvolvimento ou especialização desse sujeito particular (...) - que requer a implementação de processos de controle como planejamento, antecipação e avaliação de estratégias e/ou resultados. As experiências meta-cognitivas cumprem várias funções que são úteis na regulação de processos cognitivos. Por exemplo, um leitor que de repente sente que não está entendendo o que lê pode mudar sua estratégia de estudo, buscar informações adicionais em outro lugar, partir seu objetivo geral em sub objetivos e assim por diante. (HOUDÉ, 2004, p. 259, tradução e grifo nossos).
} 
Ambas as dimensões se influenciam na medida em que são usadas para aprimorar o estado de metacognição. Por exemplo, as experiências sobre a aplicação de uma estratégia podem levar a uma revisão do metaconhecimento. Um metaconhecimento bem empregado, leva à experiência metacognitiva de satisfação pela resolução bem-sucedida de um problema.

Já que no contexto de nosso estudo os estados metacognitivos são sobre estados cognitivos resultantes do exercício do pensar critico e, como tomamos ao longo de nosso texto, que a lógica é a principal condição de possibilidade do pensar crítico (i.e. uma condição metateórica), podemos dizer que os estados metacognitivos são os estados cognitivos sobre a lógica. Nesse sentido, a distinção entre conhecimento e experiência metacognitivas do pensar crítico, salva veritate, é uma distinção entre conhecimento e experiência sobre a lógica.

Para que não façamos uso do mesmo vocabulário das neurociências, o que pode nos induzir a uma interpretação equivocada ${ }^{12}$, estabeleceremos a distinção entre experiência da lógica e experiência na lógica ${ }^{13}$ para garantir a restrição a este domínio. O primeiro termo corresponde à experimentação das regras que compõem o sistema lógico (i.e. à capacidade de representar linguisticamente ato de experimentar [know that]). Por exemplo, saber que a regra Modus Ponens me permite inferir que se tenho P e tenho P implica Q, então tenho Q. Já a experiência na lógica corresponde à experiência de aplicação (o exercício, a execução prática [know how]) de um dado sistema lógico.

(M1) e (M3) podem ser resolvidos com a seguinte distinção: Há dois domínios do conhecimento representacional sobre o pensar crítico [know that]:

\footnotetext{
12 Um exemplo de interpretação equivocada é atribuir a lógica como conteúdo para todos os estados metacognitivos do pensar crítico. Quando a lógica é um dos domínios envolvidos na construção do pensar critico, o mais importante, de fato. Mas, apenas um deles. Outro, por exemplo, é o raciocínio cotidiano (informal).

13 À época do Tractatus, Wittgenstein disse não haver experiência possível que refute uma proposição lógica (WITTGENSTEIN, 2010. proposição 6.12222). No contexto de sua obra, isso significa que a lógica é transcendental. Para ele, havia apenas uma lógica e a experiência sobre ela era imutável, sempiterna. Além disso, sua interpretação de experiência é representacional, “de que as coisas são assim e assim" (proposição 5.552). Entretanto, anos mais tarde, Wittgenstein, orientado por uma visão pragmática da linguagem, define que o termo "lógica" se aplica a descrição das regras constitutivas dos jogos de linguagem: "porque aquilo que pertence à descrição de um jogo de linguagem pertence à lógica." (WITTGENSTEIN, 1969. §628.) Com esses argumentos, podemos criar uma distinção satisfatória entre experiência na lógica e experiência da lógica. A experiência da lógica seria o ato de experimentar (no sentido do Tractatus) as regras de um dado Jogo de Linguagem. Isto é, fazer uma descrição, uma representação proposicional de determinado conjunto de regras. A experiência na lógica seria o ato de experimentar no sentido de seguir regras de um determinado Jogo de Linguagem, neste sentido, o agente deve ser capaz de expressar a regra a qual segue, sempre que interpelado. (WITTGENSTEIN, 1994. §207-208; §232; §237). Em outras palavras, a experiência da lógica é "descrever um método de medida" e a experiência na lógica é "achar e dizer os resultados da medição", determinada por uma "certa constância nos resultados da medição" (WITTGENSTEIN, 1994, § 242).
} 
(a) $\mathrm{O}$ conhecimento dos fatos sobre o pensar crítico, i.e. fatos produzidos em decorrência do emprego do pensar crítico;

(b) fatos sobre os pressupostos do pensar crítico, i.e. fatos que formam a base representacional das competências necessárias para o uso correto do pensar crítico. Que, segundo nosso recorte, são fatos sobre a lógica.

Note que, saber sobre (a) não implica saber sobre (b) e nem saber sobre (b) implica saber sobre (a). Entretanto, como defendido ao longo do texto, é correto dizermos que para o indivíduo ser capaz de empregar o pensar crítico [know how], ele deve conhecer tanto (a), quanto (b), uma vez que conhecer (a) é identificar o estado meta-cognitivo necessário para a auto avaliação do pensar crítico e conhecer (b) é conhecer fatos sobre a lógica, onde ela é o conhecimento que fornece as condições de possibilidade para o pensar crítico.

Já a questão (P1) pode ser exemplificada elucidando-se (H1) e (H2). Ao definir seu programa de ensino da lógica, Hiller compromete-se com certos pressupostos filosóficos da lógica que não encontram em sua exposição uma definição explícita. Esses pressupostos são as questões (H1) e (H2). (H1) diz respeito a uma interpretação filosófica da dedução: Hiller entende que a dedução não deve servir somente para preservar a verdade das premissas na conclusão, mas para garantir a consistência da dedução em função das premissas. Essa interpretação é equivalente ao que apresenta Corcoran com o termo lógica teoréticotransformacional. Ela, ao contrário da lógica teorético-informacional:

\footnotetext{
Diz que a implicação é uma relação transformacional: para que um dado conjunto de premissas implique uma conclusão é necessário e suficiente que para cada transformação das premissas que as indicam como verdade, levem a conclusão também à verdade. Colocando negativamente, esta é a famosa concepção-semcontratransformação da implicação: a existência de uma relação de implicação de um conjunto de premissas a uma conclusão é a ausência de uma contratransformação, levando as premissas à verdade e a conclusão à falsidade. (CORCORAN, 1999, p. 33, tradução nossa)
}

Onde transformação é entendida por Corcoran como a substituição do conteúdo conceitual (intencional) de dada quantidade de premissas em um dado conjunto, em que este servirá de base para uma dedução. A substituição deve preservar a forma lógica das premissas e a forma e conteúdo original da conclusão. Se a transformação tornar as premissas falsas e a conclusão verdadeira, elas são proposições independentes, assim a dedução não expressa necessidade lógica. Se a transformação preservar o valor de verdade das premissas e da conclusão, dizemos que as premissas implicam necessariamente a conclusão, através da 
prática de transformações executadas no conjunto de premissas. A concordância desse conceito com o posicionamento filosófico de Hiller é provado através de seu exemplo envolvendo preconceitos cognitivos (cognitive biases):

\begin{abstract}
Os indivíduos foram apresentados com um triplo de números, "2, 4, 6", e foram solicitados a fornecer os próximos três números na sequência. $\mathrm{O}$ experimentador então os diria se seu triplo está ou não em conformidade com a regra usada para gerar a sequência. Os sujeitos do experimento de Wason e os alunos de minha classe, invariavelmente fornecem suposições como "8, 10, 12" ou "11, 13, 15", que recebem uma resposta confirmadora do experimentador ("sim, o triplo que você forneceu está em conformidade com a regra"). O truque é, naturalmente, que a regra é "qualquer sequência ascendente", então praticamente todos recebem confirmação de sua hipótese.

O que é notório no experimento de Wason é que os sujeitos apenas testam exemplos positivos, triplos que se encaixam em suas regras hipotéticas. Muito raramente eles oferecem um palpite potencialmente desconcertante, como "6, 4, 2." Isso evita que os sujeitos adivinhem a regra correta ("qualquer sequência ascendente") e permita que suas crenças incorretas persistam ("a sequência aumenta em dois cada vez"). Mais significativamente, porém, é que isso demonstra nosso preconceito para confirmar evidências. (HILLER, 2014, p. 31, tradução nossa).
\end{abstract}

Note que por preconceito cognitivo, podemos entender os casos em que as proposições são independentes. E é aqui que fazemos a passagem para (H2).

Se for possível dizer que Hiller compromete-se com uma visão teoréticotransformacional da implicação, então ele está comprometido com a consequência epistemológica dessa concepção de lógica. Tal posição entende a dedução como um esforço em criar (imaginar, explorar) novas transformações e julgá-las à luz da conclusão (CORCORAN, 1999, p. 33), e isso significa que um bom raciocínio envolve a elaboração $e$ eliminação de alternativas (HILLER, 2014, p. 31). Mas, Hiller pretende mais que uma interpretação da implicação lógica como a exploração de possibilidades. Ele tem por ênfase aproximá-la do raciocínio prático, cotidiano, dotado de propriedades não tematizadas em sistemas formais clássicos, ainda que preserve as propriedades da lógica clássica ${ }^{14}$.

Por um momento, voltemos às questões de conhecer fatos sobre o pensar crítico (a) e sobre fatos acerca dos pressupostos do pensar crítico (a lógica) (b). Conhecer somente (a) e (b) garante que o estudante seja competente em lógica? Claro que não. Devemos empregar

\footnotetext{
${ }^{14}$ Nas palavras de Hiller (2014, p. 33), essa junção chama-se bom raciocínio (good reasoning). Este conceito demanda duas habilidades chave: The first is an appreciation for the defeasibility of evidential reasoning, and the fact that in many situations the conclusions drawn are only tentatively supported. Good reasoners should always be on the lookout for unconceived alternatives, always expanding their space of possible conclusions. Related to this is the second aspect of good reasoning that defeasibility draws attention to, and that is the previously discussed role of creativity. Not only is creativity and imagination necessary in generating the initial list of relevant alternatives, it is likewise essential in coming up with new alternatives should this initial list be exhausted.
} 
uma nova divisão entre os saberes. O que antes chamamos de fatos sobre a lógica (b), agora deve ser decompostos em fatos que decorrem da aplicação da lógica $\left(b_{1}\right)$ e fatos estritamente lógicos $\left(\mathrm{b}_{2}\right)$. É o que chamamos acima de experiência na lógica e experiência da lógica, respectivamente. A primeira é amplamente apresentada por Hiller, ela é a aprendizagem do emprego da lógica em situações-problema, podendo ser estendida até o limite de decidir qual lógica deve ser preferida para resolver o problema. A segunda, como vimos, é a experiência representacional (ou conhecimento proposicional) dos princípios que governam determinado sistema lógico, e este tipo de conhecimento não é evidente nos textos de Hiller e Mulnix. Mas pretendemos esclarecê-lo.

$\mathrm{Na}$ argumentação de ambos, há um hiato entre as deduções que fazemos em um sistema lógico e o raciocínio que empregamos no curso de nosso dia a dia. Por várias razões, a lógica (clássica) opera em situações ideais, por exemplo, com fechamento dedutivo, com certas propriedades que podem ser provadas (correção, consistência, etc.). Já o raciocínio cotidiano, trabalha com crenças muitas das vezes inconsistentes, e, acima de tudo, com um tempo de execução extremamente curto. Assim, o processo de verificação de inconsistências ou a exaustão das deduções de um dado conjunto de premissas, deve ser ignorado em detrimento da demanda por respostas rápidas (WEINTRAUB, 2001, p. 3-18). E este é o ponto (H2), pois ele diz respeito a um posicionamento sobre o modo como conhecemos a lógica, i.e. de que modo a lógica se entrelaça com nossas atividades mentais, cognitivas e comportamentais com vistas a resolução de problemas cotidianos.

Para esclarecer essa aproximação, recorro às distinções de Corine Besson (2012, p. 5982). Para ela, o emprego do raciocínio cotidiano (reasoned change in view) não conflita com os princípios da lógica, apesar da diferença de pressupostos e propriedades, se tomamos o conhecimento desses princípios como proposicional (representacional) e não como disposicional (comportamental) ${ }^{15}$. Em outras palavras, a lógica não conflita com o raciocínio ordinário, se nos elementos que compõem a aprendizagem da lógica, os princípios forem ensinados a partir do "saber que" [know that], entendidos como experiência da lógica; e não no formato do "saber como" [know how], como experiência na lógica. Ou seja, aprender lógica também envolve $\left(b_{2}\right)$, especialmente se quisermos que a lógica dê suporte adequado ao good reasoning, por exemplo.

\footnotetext{
${ }^{15}$ Para uma caracterização do conhecimento da lógica como disposicional (BOGHOSSIAN, 2000, p. 229-254).
} 
Note que na medida em que o estudante adquire mais experiência da lógica, mais fatos lógicos serão considerados triviais por ele, maior será sua competência na lógica (BESSON, 2010, p. 233-268). Nesse sentido, conhecer a lógica não deve se limitar à função de preservar a verdade (ou preservar a informação) e deduzir argumentos (isto é satisfeito na experiência da lógica), mas elaborar o maior número possível de transformações (CORCORAN, 1999, p. 25-35) e, desse modo, produzir o maior número possível de fatos lógicos entre as premissas e a conclusão, aumentando o grau de conhecimento do estudante sobre a lógica da qual faz uso.

Por fim, nos resta expor (P2). Embora a discussão sobre essa questão mereça maior exposição, nos limitaremos a justificá-la como necessária para o estudo da lógica na filosofia. Como vimos, é de vital importância compreender a lógica como uma técnica que deve ser direcionada para solução de problemas práticos, pois esta é a base para o desenvolvimento do pensar crítico. Nesse sentido, faz parte do processo de identificação de um problema a ser resolvido reconhecer seu contexto e identificar qual abordagem lógica é mais adequada. A compreensão da história da lógica nos auxilia na identificação de elementos comuns entre o contexto histórico do desenvolvimento da lógica (das técnicas, dos conceitos e finalidades) e o contexto do problema a ser resolvido. Isso se torna ainda mais importante se considerarmos a pluralidade de sistemas lógicos, cada qual com sua história de aplicação bem sucedida, como por exemplo, a lógica para-consistente em programas de tomada de decisão, a lógica fuzzy para aprendizagem de máquinas, ou lógica linear para sistemas dotados de recursos dedutivos limitados, etc.

\section{CONCLUSÃO}

Há duas consequências para a leitura que fizemos. Em primeiro lugar, (P1) pôde ser exemplificado através de uma exposição do posicionamento epistemológico da lógica implícito na proposta pedagógica de Hiller. Esse tipo de interpretação é o que deve ocorrer em um contexto de ensino da lógica para a filosofia. Inicialmente, devem ser explicitadas que habilidades que se pretende desenvolver com o estudo da lógica, em seguida, quais as vantagens serão obtidas quando os estudantes se apropriarem das técnicas da lógica e, além disso, para que os estudantes entendam que toda concepção lógica pressupõe um comprometimento filosófico, seja ele de natureza epistemológica, metafísica ou até mesmo ética, devemos explicitar nosso próprio comprometimento com certa visão filosófica da lógica. 
Em segundo lugar, nossa interpretação sugere que a questão (M1) deva ser reformulada de modo que ambas as formas de conhecimento [know that $]$ e [know how], tal como definido pelo estado meta-cognitivo do pensar crítico, devam ser colocadas lado a lado em grau de importância. Tanto horizontalmente, ou seja, quando se trata do mesmo domínio teórico, quanto verticalmente, quando se trata da relação entre níveis teóricos distintos: metadomínio (filosofia da lógica) e domínio objeto (lógica prática).

Por fim, resta dizer que este é apenas um quadro possível de apresentação da lógica no contexto das práticas de ensino. Um percurso que pretende ser exequível em um ou dois semestres. Mas, que acima de sua exequibilidade esteja contida na nossa proposta o poder transformador da aprendizagem e do exercício da lógica, que visa tornar os estudantes indivíduos mais capazes de exercer individual ou coletivamente o pleno gozo de seu pensar crítico.

\section{REFERÊNCIAS}

ABRAMI, P.C.; BERNARD, R.M.; BOROKHOVSKI, E.; WADEM, A.; SURKES, M. A.; TAMIM, R.; ZHANG, D. Instructional interventions affecting critical thinking skills and dispositions: a stage 1 meta-analysis. Review of Educational Research, n.4, v.78, 2008, p. 1102-1134.

BESSON, Corine. Logical knowledge and ordinary reasoning. Philosophical studies: an international journal for philosophy in the analytic tradition, n.1, v.158, 2012, p. 59-82.

BESSON, Corine. Propositions, dispositions and logical knowledge. In: BONELLI, M.; LONGO, A. (Ed.). Quid Est Veritas? Essays in honour of Jonathan Barnes. Napoli: Bibliopolis, 2010, p. 233-268.

BOGHOSSIAN, P.; PEACOCKE, P. (Ed.). New essays on the a priori. Oxford: Oxford University Press, 2000.

CEDERBLOM, J \& PAULSEN, D.W. Critical Reasoning: understanding and criticizing arguments and theories, 6.ed. Belmont: Thomson Wadsworth, 2006.

COOK, Roy T.; Verbete: Logic. In: Dictionary of Philosophical Logic. Edinburgh: Edinburgh University Press, 2009.

CORCORAN, John. Information-theoretic logic and transformation-theoretic logic. In: Fragments of science: festschrift for Mendel Sachs. New York: World Scientific Pub.CO. 1999.

COX, Richard T. Algebra of Probable Inference. Baltimore: The Johns Hopkins University Press, 2001. 
DAVIES, M., BARNETT, R. (ed.) Palgrave handbook of critical thinking in higher education. New York: Palgrave Macmillan, 2015.

DAWSON, R. E. Critical Thinking, Scientific Thinking, and Everyday Thinking:

Metacognition about cognition. In: Academic Exchange, 2000, p. 76-83.

ELDER, Linda. Critical thinking and emotional intelligence. Inquiry: Critical Thinking Across the Disciplines, n.2, v.16, 1996, p. 35-49.

FACIONE, P. A.; FACIONE, N. C.; GIANCARLO, C. A. The disposition toward critical thinking: its character, measurement, and relationship to critical thinking skill. Journal of Informal Logic, n.1, v.20, 2000. pp.61-84.

FACIONE, Peter A. Critical thinking: what it is and why it counts? California: California Academic Press, 2007.

FACIONE, Peter A. Critical thinking: a statement of expert consensus for purposes of educational assessment and instruction. In: American philosophical association delphi research report. Millbrae: California Academic Press, 1990.

FREGE, G. O pensamento: uma investigação lógica. In: Investigações lógicas. Organização, tradução e notas de Paulo Alcoforado. Porto Alegre: EDIPUCRS, 2002.

HILLER, John. Teaching practical logic: a unifying approach. Teaching philosophy, n. 37, v. 1, 2014, p. 19-36.

HONDERICH, Ted. (ed.) The Oxford companion to philosophy. 2. ed. Oxford: Oxford University Press, 2005.

HOUDÉ, Olivier (ed.). Dictionary of cognitive science: neuroscience, psychology, artificial intelligence, linguistics, and philosophy. New York: Psychology Press, 2004.

HURLEY, Patrick J. A concise introduction to logic. Boston: Thomson Wadsworth, 2012.

IMAGUIRE, Guido; BARROSO, Cícero A. C. Lógica: os jogos da razão. Ceará: Editora Universidade Federal do Ceará, 2006.

MADDY, Penelope. A second philosophy of logic. In: RUSH, Penelope. The metaphysics of logic. Cambridge: Cambridge University Press, 2014, p. 93-108.

MORAVCSIK, Julius. Logic before Aristotle: development or birth? In: Handbook of the history of logic (volume 1) greek, indian and arabic logic. Amsterdan: Elsevier, 2004, p. 126.

MULNIX, J. W.; MULNIX, M. J. Using a Writing Portfolio Project to Teach Critical Thinking Skills. Teaching Philosophy, n.33, v.1, 2010, p. 27-54. 
MULNIX, Jennifer W. Thinking critically about critical thinking. In: Educational philosophy and theory. Oxford: Blackwell Pub., 2010, p. 1-16.

POPPER, Karl R. Conjecturas e refutações: o progresso do conhecimento científico. 4.ed. Tradução de Sérgio Bath. Brasília: Editora Universidade de Brasília, 1972.

QUITADAMO, Ian J.; FAIOLA, Celia L.; JOHNSON, James E.; KURTZ, Martha J. Community-based Inquiry Improves Critical Thinking in General Education Biology. CBELife Sciences Education. Ellensburg: Central Washington University, v.7, 2008. pp. 327 337.

SCHAAR, Maria van der. Judgement and the epistemic foundation of logic. New York: Springer, 2013.

TARSKI, Alfred. Introduction to logic and to the methodology of deductive sciences. New York: Oxford University Press, 1994.

VAUGHN, Lewis.; MACDONALD, Chris. The power of critical thinking. 2.ed. Canadense Oxford: Oxford University Press, 2010.

WEINTRAUB, Ruth. Logical knowledge. International Journal of Philosophical Studies, n. 9, v. 1, 2001, p. 3-18.

WHIMBEY, Arthur.; LOCHHEAD, Jack. Problem solving and comprehension. London: Lawrence Erlbaum Associates Inc., 1999.

WILLINGHAM, D. T. Critical thinking: why is it so hard to teach? In: DAVIES, M., BARNETT, R. Palgrave handbook of critical thinking. New York: Palgrave Macmillan, 2014.

WITTGENSTEIN, Ludwig. Da certeza. Edição bilíngue. Trad. António Fidalgo. Lisboa: Edições 70, 1969.

WITTGENSTEIN, Ludwig. Investigações filosóficas. Trad. Marcos G. Montagnoli. Petrópolis: Vozes, 1994.

WITTGENSTEIN, Ludwig. Tractatus logico-philosophicus. Trad. Luiz Henrique Lopes dos Santos. São Paulo: Edusp, 2010 [1922].

\section{Outras fontes:}

COTHRAN, Mertin. How to Teach Logic. In: https://www.memoriapress.com/articles/howteach-logic/ (acesado em 28/12/2016)

UNESCO. Declaração dos Princípios da Tolerância. Disponível em http://portal.unesco.org/en/ev.phpURL ID $=13175 \&$ URL DO=DO TOPIC\&URL_SECTION=201.html (acessado em 28/12/2016) 
OECD. Organisation for Economic Co-operation and Development. PISA, disponível em: http://www.oecd.org/pisa/data/ (acessado em 28/12/2016).

USNEWS. Ranque das melhores universidades do mundo em matemática, disponível em: http://www.usnews.com/education/best-global-universities/mathematics. (acessado em 28/12/2016).

INEP. Instituto Nacional de Estudos e Pesquisas Educacionais Anísio Teixeira. PISA, disponível em: http://portal.inep.gov.br/internacional-novo-pisa-resultados. (acessado em 28/12/2016). 